\title{
Physics not Chemistry will Solve the Cancer Problem
}

\author{
Maria Kuman* \\ Holistic Research Institute, USA \\ *Corresponding author: Maria Kuman, Holistic Research Institute, 1414 Barcelona Dr, Knoxville, TN 37923, USA
}

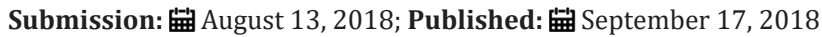

\begin{abstract}
Experimental studies show that the cells communicate between themselves fast. Since the transfer of biochemical information is slow, obviously fields are involved in these intracellular communications. It was also found that the space between the cells, which is mostly water and collagen, is very sensitive to weak external electromagnetic fields (EMFs). Another set of experiments show that the cells communicate with light and this is coherent (laser type) of light. However, the cells communicate not only with light. Japanese experiments demonstrated that the involved field responds to sound as well. It was found that the involved field is nonlinear and that not only DNA and RNA emit weak nonlinear electromagnetic field (NEMF), they respond to weak NEMF. It seems that information about the development of each cell, tissue, organ, and organism is stored not only in the chromosomes, but also in the intracellular-space liquid. Since cancer, in its last stage malignancy, is a conglomerate of strongly electrically negative cells multiplying out of control, disturbances of the NEMF of the intracellular space could be expected. But if this is the case, physics not chemistry will solve the cancer problem. The phenomenon 'phantom DNA' is also explained as imprint of the DNA field and it opens new horizons for quantum medicine.
\end{abstract}

Keywords: Fast cell communication; Cells communicate with fields (NEMF); The NEMF rules and regulates; Explaining phantom DNA

\section{Introduction}

\section{Cancer, photons and our Nonlinear Electromagnetic Field (NEMF)}

Cancer cells are negatively charged, which makes the cells to repulse each other instead of adhering to each other [1,2]. Implanted metal plates in a tissue could cause tumor formation, but experiments showed that when the plate is with nonreflecting walls, tumors are formed in $12 \%$ of the cases. However, when the plate is with reflecting walls tumors are formed in $49 \%$ of the cases [3].

Another interesting fact is: tumor cells are not found right next to the implanted plate, but at some distance from it. The tumor cells seem to be formed in the joints of the standing waves reflected from the implant. It seems like the reflected from the implant waves are like noise for transferred wave information [3]. The fact that the tumor cells were found only at the joints of stationary electromagnetic waves means that the waves are stationary. This was also found independently in the studies of Valerie Hunt [4].

\section{Experimental data [5] showed that:}

1. Based on studies of the temperature dependence of photoemission during physiological processes, it was concluded that photoemission is the basis of the body's regulating mechanisms.

2. The specter of photoemission and the statistic of photon calculations showed that the biological emission is coherent (of laser type).
3. It was found that populations of cells are effective photon accumulators. The wavelength of these photons is in the interval 350-800nm.

4. It was found that DNA is one of the major emitters of photons, which makes possible the so-called "Phantom DNA" (see section 3).

5. It was found that electromagnetic radiation with different wavelengths could influence the transfer linear ->cyclic DNA.

If the photons play such essential role in the body metabolism, it seems our science shouldn't ignore any more in its studies the super-weak human photon field, which we call aura. But the field contains more than just photons. From acupuncture we know that the acupuncture points are vortices of our EMF [6]. If so, the EMF of our body is nonlinear and we will call it nonlinear electromagnetic field (NEMF). Then the acupuncture meridians are the pathways along which the energy of our NEMF runs.

Based on nonlinear mathematical model of acupuncture meridian, the author predicted in 1983 that waves must run along the acupuncture meridians. A Hungarian scientist experimentally found the waves a year later. The author later explained in her acupuncture book [6] that our ability to react fast is done through the waves running on the surface of the body, which belong to our NEMF. Should our life depend only on the slow response of our nervous system, we would be dead long time ago. 
It was experimentally confirmed [3] that information about the development of each cell, tissue, organ, and organism is stored not only in the chromosomes, but also in the intracellular-space liquid. Some scientists, like Volpert and the Russian scientist Petuhov [3], believed before the experimental confirmation that the intracellular space must carry the field information of the volume growth. If so, the cancer cells, which have forgotten what they should build and multiply senselessly, are result of distorted information of the weak NEMF, which rules and regulates everything in the body.

The question of transport of information from one cell to another is not trivial. Gurvich investigated the field interaction between two cultures. A quartz wall separated two cellular cultures and when one of the cultures was infected with virus, the other culture also died. The transferred information from one cellular culture to another should be of the type transferred from one cell to another, i.e. involving the same type of NEMF.

Since all experiments show that the cells communicate fast and the biochemical info-transfer is slow [3], this proves that cellular cultures or cells communicate with fields. While the biochemical signals are well studied, not much is known about the field communication between the cells. One of the ways cells communicate is through photons, as concluded in point 1 based on studies of the temperature dependence of photoemission during physiological processes [5].

These and other studies showed that photoemission is the basis of the body's regulating mechanisms. Therefore, the human NEMF, to which the photons belong, rules and regulates all biochemical processes in the body [6]. But NEMF is not only photons. Japanese experiments show that our NEMF responds to sounds as well [6]. If so, we should be able to regulate the 'crazy' cellular growth of the cancerous cells by modulating the human weak NEMF (more experimental proof follows).

\section{"Phantom DNA"}

The French immunologist Jacques Benveniste [7] did research with anti-bodies in 1988. He noticed that the biological effect of his anti-bodies was still there even when the solution was so diluted that anti-bodies were no longer present. This reminded him homeopathy. He stated that the water must have the ability to memorize. Only memorizing could explain his experiment, as well as the fact that the homeopathic remedies work even when the substance is so diluted that not even one molecule of the substance is left [8].

When asked, how the water does the memorizing, Benveniste's answer was I am immunologist, not a physicist. Being a physicist, I would try to explain how the water memorizes the information of the substance that was diluted in it. The liquid water has inhomogeneous structure because only about half of the hydrogen bonds in the ice were destroyed during the process of melting. So, the liquid water is ice-clusters swimming in liquid water.

Every media, which is inhomogeneous, exhibit nonlinear properties [9] and the ice clusters present in liquid water make the liquid water strongly inhomogeneous. One of these nonlinear properties is hysteresis, which means history is important, and the fact that history is important means memorizing past events. Thus, the inhomogeneous structure of water guarantees ability to memorize.

However, if Benveniste [6] would use the word hysteresis instead of memory, when explaining that the biological effect of anti-bodies was still present when the solution was so diluted that anti-bodies were no longer present, probably it would be accepted. However, since he was not a physicist and knew nothing about hysteresis, he used the word memory, which irritated many scientists who jump on him with accusations.

The experiment of Benveniste [6] explains why the homeopathic remedies work even when the substance is so diluted that not even one molecule of the substance is left. This means that the specific vibrations of the nonlinear electromagnetic field (NEMF) of the molecules dissolved in the water are imprinted on the complicated inhomogeneous dynamic nonlinear structure of water.

The water structure is dynamic because each cluster has two layers of loosely connected water molecules. They can stay connected to the cluster or leave depending on internal or external conditions. If the conditions require these two layers to become disconnected from the cluster, another two layers of the cluster become loose and free to leave if the conditions change.

The critics of the Benveniste's idea of memorizing water didn't curb his enthusiasm for farther experimenting. Benveniste was a scientist with a vision and he claimed based on his farther experiments that biomolecules communicate with their receptors by sending low frequency electromagnetic signals, which the receptors pick up like radios tuned to specific wavelength.

He recorded these signals digitally and by playing them back in the absence of the molecules, he was reproducing the biochemical effect, as if the molecules were there. By playing the electromagnetic signal, he was able to trigger the defense response of neutrophils to kill invading cells, just as the presence of the molecules would do.

The Nobel Prize winner of year 2014 for his research on HIV virus, Luc Montagnier, found that the DNA of HIV virus, when diluted in water, created electromagnetic signal, which still existed even at very high dissolutions, when DNA molecules were no longer present. He filtered the water containing the DNA of HIV virus. After the filtration, when not even one molecule of DNA was left, the DNA signal of the HIV virus was still there, and so was its detrimental effect $[10,11]$.

\section{Cancer and the electromagnetic waves influencing the intracellular space}

Let's go back to the beginning. The cancer cells are electrically negative and for that reason they repulse each other instead of attracting each other. Let us see what is in the space between the cells, called intracellular space. It is mostly water, 5 types of collagens, elastin, fibro-lactam, glucosamine, laminin, etc. But the major components are water and collagens. It was found that the collagens are very sensitive to external EMF $[3,12]$ and so is the water. Very sensitive to external EMF are also the collagens of the bone matrix system. 
The functioning of all our organs is subordinated to the Subconscious because we don't have conscious awareness of their function It is deliberately done so because if we are solving a life-important problem, we don't want to be bothered with information about the functioning of our organs $[6,13]$. From acupuncture we know that all body organs are holographically represented on the surface of the skin: on the palms, feet, ears, (and irises of the eyes) [6]. All acupuncturists know that they could treat the sick organ by using any of these holographic representations. Based on this, we could claim that the Subconscious works on the holographic principle.

Holographic images are three-dimensional and only laser waves can create them. As said in point 2 , the photoemission of the human body was found to be of laser type. If only laser waves can create holographic images, our Subconscious, which works on the holographic principle with holographic images, must rule and regulate all organs through the coherent (laser type of) waves of our weak NEMF.

Another proof of this is the fact that Russian scientists studied a person that can see with blindfolded eyes. He sees with his mind [14] and he sees the object as three-dimensional holographic image. This allows him to see the backside of the object, and he could even see the object when it is hidden behind a screen. Since only nonlinear waves can pass through a screen, this is another proof that the laser waves of our NEMF are nonlinear.

Since the Subconscious rules the functioning of all organs on the holographic principle, the weak NEMF that rules and regulates the functioning of all organs and the rest of the body must be located in the Subconscious. Since it operates with waves, it must operate as a Quantum Computer, which from the Subconscious rules and regulates everything in the body. Our Mind is a part of our NEMF - it is not product of the brain as some scientists think. Proof of it is the seeing with the Mind [14], which is holographic, and can be done only with waves.

\section{Slow biochemical signals or fast EMF signals}

As said, the biochemical info-transfer is slow, but data show [3] that cells communicate fast, which means that fields are involved. If the biochemical transfers are slow, we can expect our conscious mind, which works on biochemical basis to be slow. The Subconscious that work on holographic principle with waves is expected to be much faster. Indeed, hypnosis, which put the Conscious to sleep to allow access to the Subconscious, shows that our Subconscious has a very high speed of computation.

Based on this we can claim that our Conscious, which works on the basis of biochemical transfers, is our digital computer. It is relatively slow because the biochemical transfers are slow. Our Subconscious, which works on the basis of waves, is our Quantum Computer. No wonder it is very fast because the waves propagate fast. Since the Subconscious work on the holographic principle and holographic record is compact, our Subconscious (our Quantum Computer) has extremely large storage capability.
If our Subconscious rules and regulates the functioning of all organs and everything else in the body (including the cellular growth) and this is done through the waves of our NEMF, we can expect the cancer cells, which multiply senselessly, to have distorted regulating mechanism, i.e. distorted NEMF. If so, we can expect to be able to restore the normal functioning of the cancer cells by restoring the regulating NEMF.

Bissel postulated that the interaction between the cells must be retroactive [3]. I fully agree with him because according to the nonlinear theory reverse connection is absolutely necessary for every self-organized system. This means that not only will the intracellular media influence the cells, but also the cells will influence the intra-cellular media. For this reason, the DNA not only emits photons, it is sensitive to the photons of our NEMF, as well as to acoustic frequencies [3].

This means that the information, which NEMF of the intracellular space carries, is capable of influencing the DNA and RNA inside the cells, which are long chains and are conformationally labile. This conformational instability allows recording of NEMF information with one or another type of configuration [3]. If so, NEMF changes in the intracellular media could induce damage of the cellular DNA. However, the opposite is also true- if a virus has damaged the cellular DNA, this would induce changes in the intracellular media.

We don't need to find out in each particular case, from where the damage came from inside the cell or from outside. We should just restore the NEMF and this would restore the normal functioning of the cancerous cell, which have escaped the regulation because of distorted NEMF. Even when the DNA structure of the cells has been changed by virus (which made the cellular division abnormal), restored regulation by restoring the normal NEMF in the intracellular space should make the cancerous cells normal.

\section{Cancer and microwave radiation}

It was found [3] that water solutions have resonance frequency of absorption in the microwave diapason. For DNA and RNA water solutions the diapason of resonance microwave absorption was 2-9GHz. It was also found that erythrocytes have resonance microwave absorption in the band $35-40 \mathrm{GHz}$. These facts raise a red flag: the microwaved food, as well as our cellular phones and our digital TV may not be harmless. Many years ago the Russian scientists claimed based on their studies that microwaves are not safe and cause cancer.

\section{Conclusion}

I hope I have convinced you that physics has important role to play in the fight against cancer, but this is just the beginning. Physicists need to step in and come with the right tools (equipment) to fight cancer with appropriate type of NEMF. I am certain that we are going to win the battle with cancer because all biochemical transformations in the body are ruled and regulated by our NEMF. We 
just need to learn how to do the necessary correction in the regulating mechanism, which will bring back the health.

\section{References}

1. Kuman M (1993) What everybody needs to know about chronic pain chronic diseases, and cancer. Health and Happiness Books.

2. Kuman M (2018) International Journal of Complimentary and Alternative Medicine 11(6).

3. Garyaev P (1994) Kvantovoi genom (quantum genom). Moscow, Russia.

4. www.biofieldenergy.org

5. Nagle W, Popp FA (1983) Cytobios. 37: 45-62.

6. Kuman M (1997) Modern aspects of ancient acupuncture. Health and Happiness Books.

7. Benveniste J (1988) Nature. 333: 816-818.
8. Thomas I (2000) Medical hypothesis. 54: 33-39.

9. Kuman M (1988) Organic polymer conductivity -A nonlinear approach. Synthetic Metals 27: A89.

10. Montagnier L (2010) Homeopathic signals from DNA. Science and Society $48: 36-39$.

11. Montagnier L (2010) Electromagnetic signals from DNA. Science and Society 48: 40-43.

12. Kuman M (2018) Cancer is a lost control over the cellular growth caused by stress. Advances in Complimentary and Alternative Medicine 1(4): $1-2$.

13. Kuman M (2017) The acupuncture meridians are wave sensitive. International Journal of Integrated Medicine 42(2): 97-112.

14. Kuman M (2018) Holographic (Quantum) way of seeing, hearing and memorizing and their use for diagnosis. Research in Medicine and Engineering Science 5(3): 1-4.

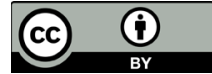

Creative Commons Attribution 4.0 International License

For possible submissions Click Here

\section{Submit Article}

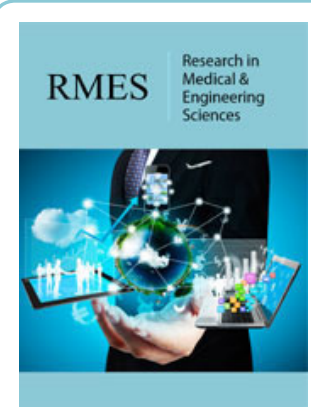

\section{Research in Medical \& Engineering Sciences}

\section{Benefits of Publishing with us}

- High-level peer review and editorial services

- Freely accessible online immediately upon publication

- Authors retain the copyright to their work

- Licensing it under a Creative Commons license

- Visibility through different online platforms 